\title{
Lasing in GaN microdisks pivoted on $\mathrm{Si}$
}

\author{
H. W. Choi, ${ }^{a}$ K. N. Hui, and P. T. Lai \\ Department of Electrical and Electronic Engineering, University of Hong Kong, Hong Kong \\ P. Chen, X. H. Zhang, S. Tripathy, J. H. Teng, and S. J. Chua \\ Institute of Materials Research and Engineering, Singapore 117602, Singapore
}

(Received 6 July 2006; accepted 2 September 2006; published online 20 November 2006)

\begin{abstract}
Arrays of pivoted $\mathrm{GaN}$ microdisks have been fabricated on a GaN/Si material by a combination of dry and wet etching. The Si material beneath the GaN microdisks is removed by wet etching, leaving behind a fine pillar to support the disks. Raman spectroscopy reveals substantial strain relaxation in these structures. Resonant modes, corresponding to whispering gallery modes, are observed in the photoluminescence spectra. Stimulated emission is achieved at higher optical pumping intensities. (C) 2006 American Institute of Physics. [DOI: 10.1063/1.2392673]
\end{abstract}

Insertion of mirrors on either side of an optical cavity is crucial towards forming resonant cavity devices. In the GaN/ sapphire system, ${ }^{1,2}$ there exists a considerable difficulty in inserting a mirror at the lower end to form an optical cavity. One of the common techniques is the growth of pairs of $\mathrm{AlN} / \mathrm{GaN}$ layers acting as reflector stacks. ${ }^{3,4}$ Such growth sequences are difficult to control, and the GaN material grown on top of the stack is often mediocre. Laser lift-off offers a way to expose the lower GaN surface for the deposition of dielectric Bragg reflector stacks. ${ }^{5}$ Nevertheless, the process is hardly suitable for mass production.

A method of forming pivoted GaN microdisks is proposed in this work using a $\mathrm{GaN} / \mathrm{Si}$ material, enabling exposure of the bottom GaN surface. Due to a large lattice mismatch between $\mathrm{GaN}$ and $\mathrm{Si}$, cracking is almost inevitable during growth, making it unsuitable for conventional broadarea light emitting diodes. Nevertheless, high quality GaNon-Si films have been demonstrated recently. ${ }^{6,7}$ With an everdecreasing crack density, it is possible to fit a reasonable number of microscale devices between the cracks-this is now being exploited to fabricate pivoted microdisk arrays. The fabrication sequences are simple and mass manufacturable: GaN/Si samples are photoresist coated and patterned, and the $20 \mu \mathrm{m}$ microdisks are subsequently transferred onto the $1 \mu \mathrm{m}$ thick $\mathrm{GaN}$ layer structures by dry etching using $\mathrm{Cl}_{2} / \mathrm{BCl}_{3}$ gases, which consisted of a series of multiquantum wells (MQWs). Intermediate AlGaN/AlN buffer layers were used to reduce the crack density in these samples. The sample is dipped in a solution containing $\mathrm{HNO}_{3}, \mathrm{H}_{2} \mathrm{O}$, and $\mathrm{NH}_{4} \mathrm{~F}$, an isotropic wet etchant which creates an undercut as it removes the $\mathrm{Si}$ surrounding the $\mathrm{GaN}$ microdisks. The wet etching is allowed to continue until a tiny pedestal of $\mathrm{Si}$ remains; this pedestal supports the microdisk and provides a pathway for current injection. Due to the isotropic nature of the wet etchant, the pedestal tapers outwards so that the contact interface between $\mathrm{GaN}$ and $\mathrm{Si}$ is much smaller than the base of the Si pedestal, as shown in the cross-sectional scanning electron microscopy (SEM) image in Fig. 1(a). A microdisk array is illustrated in Fig. 1(b). This represents a convenient method of forming microdisks as compared to other approaches reported. ${ }^{8}$

\footnotetext{
a) Author to whom correspondence should be addressed; FAX: (852)
} 25598738; electronic mail: hwchoi@eee.hku.hk
Using this approach, the lower GaN surface has been exposed, which can potentially be mirror coated. GaN grown on Si substrates are known to be highly strained, giving rise to crack formation. The spacing between cracks in our samples is measured at $50-100 \mu \mathrm{m}$. This tensile strain is also exhibited in the Raman spectrum plotted in Fig. 2, whereby an Ar ion laser beam at $514.5 \mathrm{~nm}$ was focused to a spot of $1 \mu \mathrm{m}$ diameter and the signal was collected and analyzed by JY-T64000 liquid-nitrogen-cooled triple grating spectrometer in the $z\left(x_{-}\right) \bar{z}$ backscattering configuration, giving a spectral resolution of $0.2 \mathrm{~cm}^{-1}$. The focused laser light was excited at a spot away from the cracks on the as-grown sample, and the $E_{2}$ (high) and $A_{1} \mathrm{LO}$ phonon modes at 564.5 and $735.5 \mathrm{~cm}^{-1}$ were observed, together with $520.5 \mathrm{~cm}^{-1}$ phonon frequency due to the underlying $\mathrm{Si}$. The Raman excitation power was kept very low to avoid any red thermal shift. In the case of GaN, usually the $E_{2}$ (high) and $A_{1}(\mathrm{LO})$ modes are observed in the $z(x x) \bar{z}$ geometry, whereas only $E_{2}($ high) appears in the Raman spectrum in the $z(x y) \bar{z}$ configuration. In our case, the outgoing scattered light was not analyzed for its polarization and we have recorded both $E_{2}$ (high) and $A_{1}(\mathrm{LO})$ phonons. The $E_{2}$ (high) Raman peak is generally used to estimate the in-plane strain in $\mathrm{GaN} .{ }^{9,10} \mathrm{As}$ a reference, the $E_{2}$ (high) phonon peak measured from a $400 \mu \mathrm{m}$ thick freestanding strain-free $\mathrm{GaN}$ was observed at $567.5 \mathrm{~cm}^{-1}$. The in-plane tensile stress can be evaluated from the relation $\Delta \omega=4.3 \sigma_{x x} \mathrm{~cm}^{-1} \mathrm{GPa}^{-1}$ giving a value of $0.70 \mathrm{GPa}$ on the as-grown GaN/Si wafer. ${ }^{11} \mathrm{Next}$, the Raman signal was collected from a spot halfway between the center and edge of a microdisk. This time the $A_{1}(\mathrm{TO}), E_{1}(\mathrm{TO})$, $E_{2}\left(\right.$ high), and $A_{1}(\mathrm{LO})$ phonon modes were clearly recorded at $534.2,561.8,567.6$, and $738.3 \mathrm{~cm}^{-1}$, respectively. Due to the Raman intensity enhancement related to the Si-substrate

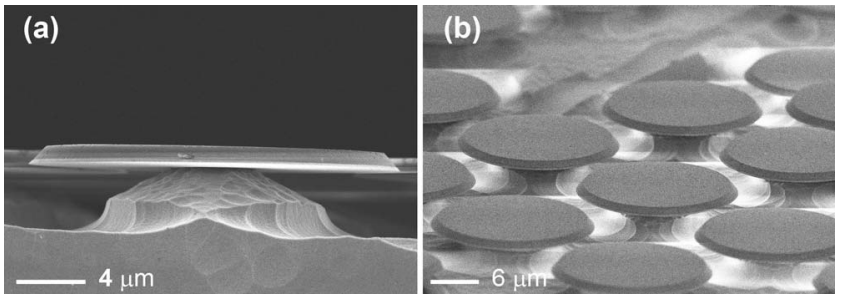

FIG. 1. SEM images showing (a) the cross section of a single pivoted microdisk and (b) a microdisk array. 


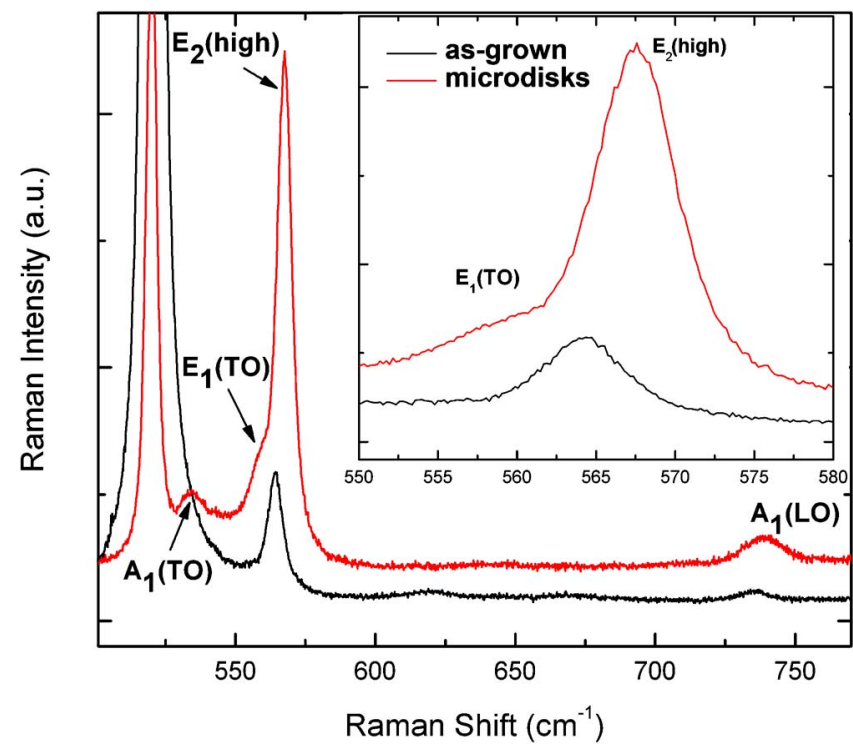

FIG. 2. (Color online) Raman spectrum of as-grown and microdisk samples. Enlarged views of the $E_{1}(\mathrm{TO})$ and $E_{2}($ high) modes are shown in the inset.

removal, we could detect all other weak Raman active phonons such as the $A_{1}(\mathrm{TO})$ and $E_{1}(\mathrm{TO})$ from the pivoted microdisk $\mathrm{GaN}$ structures. This is partly due to the deviation from a perfect backscattering geometry in the microdisks. As compared to the Raman spectrum of the as-grown $\mathrm{GaN} / \mathrm{Si}$, a large increase in the Raman scattering efficiency is clearly observed from the microdisk structures. This is due to the more efficient coupling and multiple scattering of light due to the presence of an air gap beneath the GaN surface that offers a large dielectric contrast. As we are probing the whole GaN crystal during such visible Raman excitation, the intensity enhancement also causes the much weaker modes to appear clearly in the Raman spectra. The $E_{2}$ (high) phonon peak from the pivoted microdisk structures coincides with that of freestanding GaN, signifying substantial strain relaxation in the microdisks. However, a strain gradient is observed from a mapping of the Raman signal across the disk. Typical Raman line scans with a lateral resolution less than $1.0 \mu \mathrm{m}$ show that the $E_{2}($ high) phonon peak ranges from 567.2 to $568.1 \mathrm{~cm}^{-1}$ along the microdisk. The Raman peak shows a slight blueshift $\left(568.7-568.1 \mathrm{~cm}^{-1}\right)$ near the anchor points of the microdisk (near the underlying Si pillar) and then a gradual redshift toward the edge. Such a variation of the peak shift results from the residual bending stress in the microdisk since it is only supported in the center with a narrow pedestal. Typical cantilever beams and bridges show similar variations of compressive to tensile bending stress. The presence of intermediate AlGaN and AlN buffer layers in the whole structure introduces a vertical gradient stress, and as a result a compressive stress is observed from Raman peak shifts near the anchor points. Away from these positions, the tensile bending stress further compensates the Raman peak shift, and we observe nearly stress-free values for the $E_{2}$ peak positions.

The morphology of the top and bottom GaN surfaces is evaluated. The top surface can be easily imaged with an atomic force microscopy (AFM) scan. Surface steps and void defects are clearly observed, and the material quality is inferior to GaN grown in sapphire. Nevertheless, the surface is optically smooth with a rms roughness of $1.49 \mathrm{~nm}$. In order Downloaded 20 Apr 2007 to 147.8.143.135. Redistribution subject
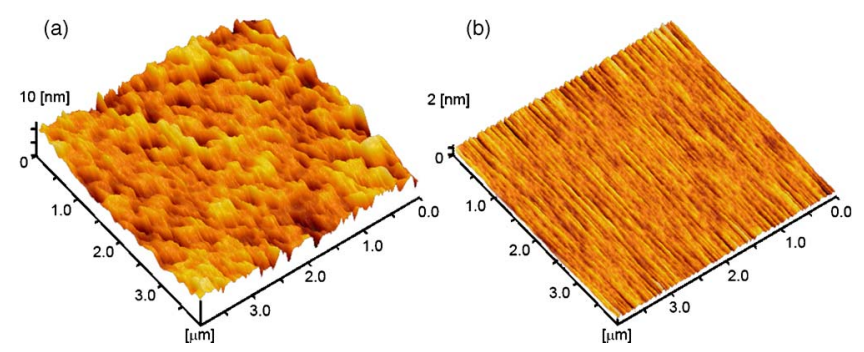

FIG. 3. (Color online) AFM three-dimensional images of the (a) top and (b) bottom surfaces of the microdisks.

to scan the lower surface, a $\mathrm{GaN} / \mathrm{Si}$ sample was epoxy bonded to the glass substrate, and this assembly was immersed in a $\mathrm{Si}$ etchant for $3 \mathrm{~h}$ to completely separate the $\mathrm{GaN}$ microdisks from the $\mathrm{Si}$ substrate. The microdisks remain bonded to glass, exposing the lower face of the disks for AFM scanning, as illustrated in Fig. 3(b). Surprisingly, this surface was even smoother than the top surface with a rms roughness of $0.2 \mathrm{~nm}$.

The optical properties of the microdisks were probed by time-integrated photoluminescence with the excitation of $150 \mathrm{fs}$ pulses at $325 \mathrm{~nm}$ from an optical parametric amplifier, which was driven by a $1 \mathrm{kHz}$ Ti:sapphire regenerative amplifier. The beam was incident at $45^{\circ}$ to the sample surface cooled to $4.3 \mathrm{~K}$, with a spot diameter of around $300 \mu \mathrm{m}$. The photoluminescence (PL) signal was dispersed in a $50 \mathrm{~cm}$ monochromator and detected by a photomultiplier and standard lock-in amplification. The PL spectra for the as-grown and microdisk samples at low excitation energies are shown in Fig. 4(a) and are noted to consist of two peaks at $\sim 360$ and $\sim 400 \mathrm{~nm}$, corresponding to the emissions from $\mathrm{GaN}$ and InGaN/GaN MQWs, respectively. Additionally, periodic resonant modes can be observed in the spectrum obtained from the microdisks, with a mode spacing of about $1.1 \mathrm{~nm}$. This can be attributed to whispering gallery mode (WGM) oscillations, predicted by the relation $\Delta \lambda_{\mathrm{WG}}=\lambda^{2} / 2 \pi R n{ }^{12}$

With increasing excitation intensities nonlinear increases in PL intensities of the $365 \mathrm{~nm}$ peak were observed beyond threshold condition, as depicted in Fig. 4(b). Lasing occuring at the GaN peak rather than the InGaN peak indicates that the quality of the MQWs is inferior to the underlying GaN layer. A plot of integrated PL intensities is also shown in the inset of the same figure. Such nonlinear increase in emission intensities is a characteristic of stimulated emission. Although lasing as a result of a vertical cavity cannot be ruled out, it is more likely to be explained by the planar microdisk cavity as the top and bottom surfaces of the disks are not mirror coated. The GaN/air interface reflects only $20 \%$ of light, making optical confinement highly inefficient. On the other hand, the microdisk structure strongly confines the WGM in the disk active layer by total internal reflection at semiconductor/air boundaries. ${ }^{13}$ From the PL spectrum, a $Q$ factor of 80 was evaluated. Although the $Q$ factor is relatively low for microcavity devices (which could be due to the inclined sidewall), it serves as proof-of-concept demonstration for $\mathrm{GaN} / \mathrm{Si}$ materials in achieving stimulated emission. Although the material quality of $\mathrm{GaN} / \mathrm{Si}$ falls short of their counterparts on sapphire, Si substrates provide numerous major advantage over sapphire. In order to fully implement a resonant cavity device, improvement in the $Q$ factor with the aid of mirror coatings and a reduction of microdisk dimensions to take advantage of the Purcell effect, ${ }^{14}$ together to AIP license or copyright, see http://apl.aip.org/apl/copyright.jsp 

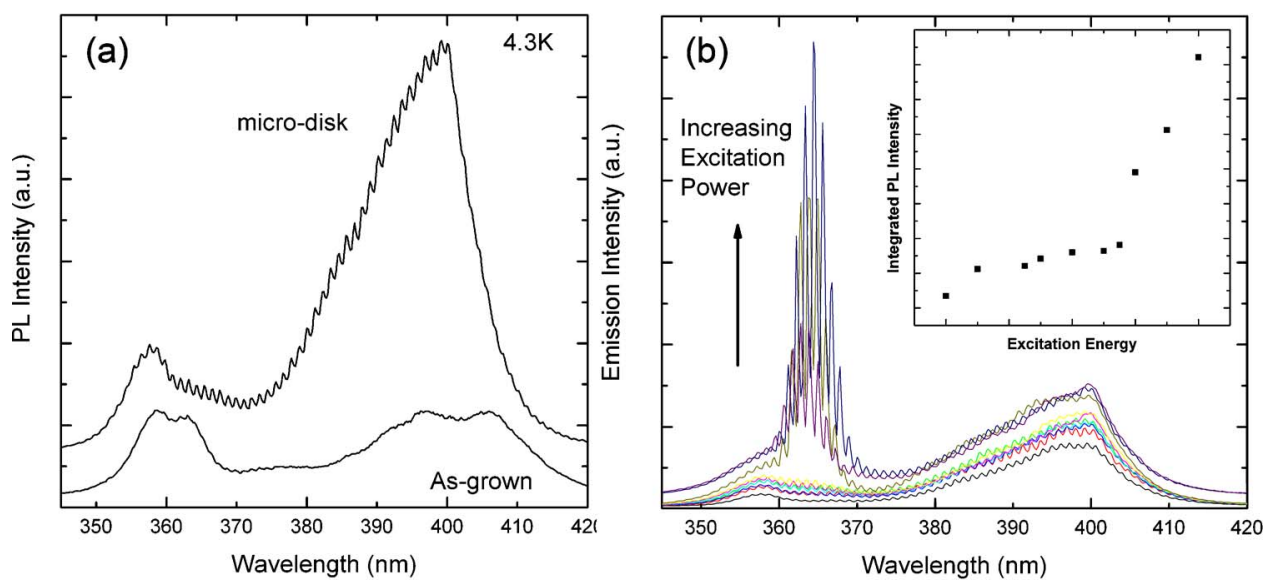

FIG. 4. (Color online) (a) PL spectrum of the as-grown and microdisk samples. (b) PL spectrum of the microdisk at increasing excitation powers. A plot of integrated PL intensity vs excitation energy is shown in the inset.

with ever-improving GaN/Si material quality, will enable the realization of optical cavity devices using GaN/Si material.

$\mathrm{GaN}$ microdisks pivoted on $\mathrm{Si}$ substrate are demonstrated, enabling optical confinement via vertical cavity modes or whispering gallery modes. Besides the observation of resonant modes, lasing action has been achieved by optical pumping, confirming the quality of the optical cavity. Current-injection lasing is promising as both surfaces of the microdisks are accessible for mirror coating, together with the fact that the complete structure is vertically conducting. We anticipate that this technique opens up a range of possibilities in GaN-based resonant cavity devices and brings us a great step towards realizing the elusive $\mathrm{GaN}$ vertical cavity surface emitting laser.

${ }^{1}$ P. Kung, C. J. Sun, A. Saxler, H. Ohsato, and M. Razeghi, J. Appl. Phys. 75, 4515 (1994).

${ }^{2}$ M. E. Lin, S. Strite, A. Agarwal, A. Salvador, G. L. Zhou, N. Teraguchi, A. Rockette, and H. Markoc, Appl. Phys. Lett. 62, 702 (1993).

${ }^{3}$ H. M. Ng, T. D. Moustakas, and S. N. G. Chu, Appl. Phys. Lett. 76, 2818 (2000).
${ }^{4}$ C. C. Kao, Y. C. Peng, H. H. Yao, J. Y. Tsai, Y. H. Chang, J. T. Chu, H. W. Huang, T. T. Kao, T. C. Lu, H. C. Kuo, and S. C. Wang, Appl. Phys. Lett. 87, 081105 (2005).

${ }^{5}$ R. W. Martin, P. R. Edwards, H. S. Kim, K. S. Kim, T. Kim, I. M. Watson, M. D. Dawson, Y. Cho, T. Sands, and N. W. Cheung, Appl. Phys. Lett. 79, 3029 (2001).

${ }^{6}$ F. Schulze, A. Dadgar, J. Blasing, A. Diez, and A. Krost, Appl. Phys. Lett. 88, 121114 (2006).

${ }^{7}$ J. M. Bethoux, P. Vennegues, F. Natali, E. Feltin, O. Tottereau, G. Nataf, P. de Mierry, and F. Semond, J. Appl. Phys. 94, 6499 (2003).

${ }^{8}$ R. Sharma, E. D. Haberer, C. Meier, E. L. Hu, and S. Nakamura, Appl. Phys. Lett. 87, 051107 (2005).

${ }^{9}$ S. Tripathy, S. J. Chua, P. Chen, and Z. L. Miao, J. Appl. Phys. 92, 3503 (2002).

${ }^{10}$ F. Demangeot, J. Frandon, M. A. Renucci, O. Briot, B. Gil, and R. L. Aulumbard, Solid State Commun. 100, 207 (1996).

${ }^{11}$ L. S. Wang, K. Y. Zang, S. Tripathy, and S. J Chua, Appl. Phys. Lett. 85, 5881 (2004).

${ }^{12}$ H. W. Choi, C. W. Jeon, M. D. Martin, P. R. Edwards, R. W. Martin, and S. Tripathy, J. Appl. Phys. 93, 5978 (2003).

${ }^{13}$ T. Baba and D. Sano, IEEE J. Sel. Top. Quantum Electron. 9, 1340 (2003).

${ }^{14}$ E. M. Purcell, Phys. Rev. 69, 681 (1946). 